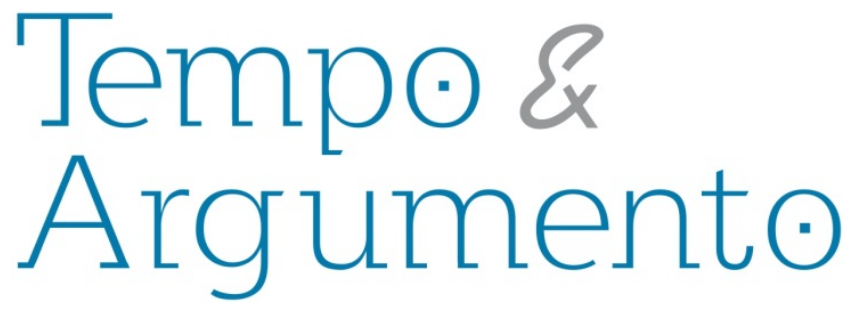

\title{
Résistance et genre dans les archives des dictatures militaires du Cône Sud
}

\begin{abstract}
Résumé
Cet article présente un réflexion sur les archives qui gardent les fonds sur les dictatures dans les pays du Cone Sul (Argentine, Bolivia, Brésil, Chili, Paraguay et Uruguay), qui ont été explorés au Projet Genre, Féminismes et Dictatures, par le Laboratoire d'Études de Genre et Histoire de I'Université de Fédérale de Santa Catarina. II essaye de montrer comment on peut trouver des perspectives de genre dans des fonds qui ont originalement eu comme objectif la répression aux mouvements politiques de résistance aux dictatures, ou qui se sont constitués justement dans l'effort de mémoire de cette résistance, mais qui n'ont pas mis en cause spécifiquement les questions de genre. La réflexion a pour objectif de montrer que le genre est un aspect important dans la constitution de discours de résistance dans ces pays.
\end{abstract}

Mots clefs: Genre. Dictature. Cone Sul. Archives. Mémoire.

\section{Pour citer cet article:}

WOLFF, Cristina Scheibe. Résistance et genre dans les archives des dictatures militaires du

Cône Sud. Revista Tempo e Argumento. Florianópolis, v. 5, n.9, jan./jun. 2013. p. 433 - 450.

\section{DOI: $10.5965 / 2175180305092013433$}

http://dx.doi.org/10.5965/2175180305092013433 


\section{Resistence and gender in the archives of the Cone Sul military dictatorships}

\begin{abstract}
This article presents a reflection on the archives that hold collections on dictatorships in the countries of the so called Cone Sul (Argentina, Bolivia, Brazil, Chile, Paraguay and Uruguay) which have been explored in the Project Gender, Feminisms and Dictatorships by the Laboratory for the Study of Gender and History of the Federal University of Santa Catarina. It aims to show how one can find gender perspectives in collections that originally had as their main purpose the repression against political movements of resistance to dictatorships, or that were constituted precisely in the memory effort of this resistance, but that did not problematized particularly gender issues. The reflection is intended to show that gender is an important aspect in the constitution of resistance discourses in these countries
\end{abstract}

Keywords: Gender. Dictatorship. Cone Sul. Archives. Memory.

\section{Resistência e gênero nos arquivos das ditaduras militares do Cone Sul}

\begin{abstract}
Resumo
Este artigo apresenta uma reflexão sobre os arquivos que guardam acervos sobre as ditaduras nos países do Cone Sul (Argentina, Bolívia, Brasil, Chile, Paraguai e Uruguai) que tem sido explorados no Projeto Gênero, Feminismos e Ditaduras pelo Laboratório de Estudos de Gênero e História da Universidade Federal de Santa Catarina. Busca mostrar como se pode encontrar perspectivas de gênero em acervos que originalmente tiveram como principal propósito a repressão aos movimentos políticos de resistência às ditaduras, ou que se constituíram justamente no esforço de memória desta resistência, mas que não problematizaram especialmente as questões de gênero. A reflexão pretende mostrar que o gênero é um aspecto importante na constituição dos discursos da resistência nestes países.
\end{abstract}

Palavras-chave: Gênero. Ditadura. Cone Sul. Arquivos. Memória. 
Les réflexions que je vais présenter aujourd'hui sont le résultat d'un projet collectif de recherche qui est en réalité la combinaison de deux recherches principales : mouvements de femmes et féminismes en temps de dictature militaire dans le cône Sud (1964-1989), coordonné par Joana Maria Pedro ${ }^{1}$ et le genre de la résistance dans la lutte contre les dictatures militaires du cône Sud (1964-1989) coordonné par Cristina Scheibe Wolff. ${ }^{2}$ À partir de ces projets, plusieurs étudiants de licence, de master, de doctorat et de post doctorat ont proposé des projets qui s'y insèrent, au sein du laboratoire d'étude du genre et d'histoire. En mai 2009, nous avons organisé le colloque international Genre, Féminisme et Dictatures dans le cône Sud, avec la participation de conférenciers des divers pays du cône Sud, et plus de 300 communications furent présentées sur ce thème. ${ }^{3}$ De plus, le livre Resistências, gênero e feminismos contra as ditaduras no Cone Sul a été récemment édité, il réunit des travaux réalisés par l'équipe du projet. ${ }^{4}$ Comme les projets ont des problématiques de recherche, des objectifs et une méthodologie qui se croise, tout comme des cadres géographique et chronologique semblables, l'équipe de recherches a travaillé en groupe dans toutes les activités de recherche. Nous avons ainsi parcouru ensemble de nombreuses archives et d'autres lieux de mémoire dans ces divers pays. Je vais essayer de présenter un petit panorama de ces espaces, afin d'éclaircir les problèmes qu'ils présentent, et leurs potentialités, à partir d'une perspective centrée sur le genre.

Pendant les décennies des années 1960 et 1970, plus ou moins en même temps, l'Argentine, la Bolivie, le Brésil, le Chili, le Paraguay et l'Uruguay ont connu des régimes militaires avec des caractéristiques similaires. La plus grande justification utilisée pour légitimer ces régimes d'exception fut la lutte contre le communisme, qui, en ces temps de

\footnotetext{
${ }^{1}$ Ce projet a été financé par le CNPq (bourse productivité $1 \mathrm{C}$ ), en plus d'aide de recherche et d'obtenir des ressources de la FAPESC.

${ }^{2}$ Projet financé par le CNPq (bourse productivité 1D), aide de recherche et bourse d'initiation scientifique.

${ }^{3}$ Ce colloque a produit les annales électroniques, disponibles sur : http://www.coloquioconesul.ufsc.br/ e o livro PEDRO, Joana Maria e WOLFF, Cristina Scheibe. Gênero, Feminismos e Ditaduras no Cone Sul. Florianópolis: Editora Mulheres, 2010. Disponível em formato pdf no site: http://www.ieg.ufsc.br/livros_eletronicos.php

${ }^{4}$ PEDRO, Joana Maria; WOLFF, Cristina Scheibe e VEIGA, Ana Maria. (org.) Resistências, gênero e feminimos contra as ditaduras no Cone Sul. Florianópolis: Mulheres, 2011.
} 

droits de I'homme et de résistance et de défense de la citoyenneté ont commencé à émerger. Des groupes de l'église catholique, liés à ce que l'on appelle la Théologie de la Libération, ont abrité des groupes de personnes avec des positions de gauche et ont promu des organisations communautaires et des initiatives pastorales, qui furent très importantes à ce moment-là, notamment dans le développement des actions en faveur des droits de l'homme.

C'est aussi à cette période que se développe en Amérique du Sud et dans le monde entier la «Nouvelle Gauche », inspirée principalement de la Révolution Cubaine et de la Révolution Chinoise, qui questionnaient les orientations des partis communistes alignés sous la position de l' Union des Républiques Socialistes Soviétiques . Les groupes de la Nouvelle Gauche se sont organisés en ayant l'espoir que les mouvements qui commenceraient avec de petits groupes d'avant-gardes pourraient s'étendre à d'autres couches sociales, comme cela s'était produit à Cuba dans les années 50, ou en croyant dans la rébellion populaire, comme celle qui avait eu lieu en Chine.

Cette période a aussi été marquée, dans le monde occidental, par la seconde vague du Mouvement Féministe. Malgré les dictatures, la contre-culture et le féminisme ont été importants dans la formation de la subjectivité de la génération des jeunes de ces différents pays.

La doctrine de la Sécurité Nationale a été élaborée à partir de la politique établie par le président américain Harry Truman, à partir de 1947. Cette doctrine considérait le communisme et les mouvements sociaux de gauche comme des menaces à la sécurité interne des États-Unis, et, à partir de la révolution Cubaine, elle a commencé à inclure tous les pays d'Amérique latine. Pour cela, les États-Unis ont commencé à financer les équipements et les entraînements des forces de police et des forces armées des divers 
ce qui a été appelée la guerre de renseignements, la guerre contre-révolutionnaire. Cette stratégie, également nommée guerre anti subversive, fut surtout développée à partir des expériences françaises dans les guerres d'Indochine, du Vietnam et d'Algérie. Elle fut diffusée à travers des « cours » et à travers la présence de conseillers militaires français, au Brésil, en Argentine, et surtout à travers l'école des Amériques au Panama, parrainé par les États-Unis, par laquelle des dizaines de milliers de militaires latino-américains sont passés.

Cette stratégie cherchait à recueillir toute l'information possible sur les organisations responsables de la « révolution », en comprenant réellement leur structure. L'objectif était de découvrir qui étaient les militants et l'organigramme de l'organisation. C'est plus ou moins ce que la police politique brésilienne a fait. En 1974, le DEOPS de l'État de Sao Paulo a distribué aux autres États un dossier dactylographié, contenant la structure générale des organisations de la gauche armée, et tout type d'information sur chacune d'entre elles : date de fondation, principaux leaders (nom et nom de code), principales actions, principes et idéologies. Pour obtenir ces informations, le principal moyen utilisé était l'emprisonnement des militants, et éventuellement des proches du militant, et leur interrogatoire, en utilisant la torture pour s'assurer que la personne parle.

Cette méthode, avec des variations, a été utilisée dans plusieurs pays du cône Sud dans lesquelles nous réalisons notre recherche. Et elle a produit des archives très sombres, qui ne sont pas toujours accessibles. Au Brésil, malgré la polémique actuelle pour l'ouverture de toutes les archives de cette période, puisqu'une partie d'entre eux n'ont pas été débloquées pour la consultation publique, I'historien Carlos Fico a argumenté que la partie réellement occulte de ses archives doit être insignifiante, compte tenu de la quantité déjà disponible. La collection Brasil Nunca Mais, disponible en consultation à l'archive Edgard Leuenroth, à I'université de Campinas, SP, (

\footnotetext{
${ }^{5}$ FICO, Carlos. Como eles agiam: os subterrâneos da ditadura militar: espionagem e polícia política. Rio de Janeiro: Record, 2001.
} 
http://segall.ifch.unicamp.br/site_ael/) en est un exemple. Selon la direction de cette archive:

\begin{abstract}
"le projet Brasil Nunca Mais a été conçu par Dom Paulo Evaristo Arns, alors assesseur du cardinal archevêque de São Paulo et par le Révérend Jaime Wright, qui était alors pasteur de l'Église Presbytérienne Unie. La collection réunit des copies de plus de 700 procès des prisonniers politiques brésiliens de la période de la dictature militaire, de 1964 à 1985, reproduits à partir des archives du Tribunal Militaire Supérieur et du Tribunal Suprême Fédéral. Elle contient des informations sur les partis et organisations visés par la répression tout comme des études sur la torture au Brésil et dans le monde. La collection présente des sections annexes, avec 10170 documents reproduits, relatifs à la documentation qui se trouvait en possession des militants politiques et annexée au procès comme " preuve de subversion ». La collection s'étend sur la période 1964 à 1979 et peut être reproduite.

(http://segall.ifch.unicamp.br/site_ael/index.php?option=com_content\&v iew=article\&id=107\&Itemid=90, consultée le 28/03/2012).
\end{abstract}

Cette collection a été constituée de manière sui generis: les dossiers de procédure et les documents qui suivent le cours de la justice étaient seulement disponibles pour les avocats des prisonniers. Ceux-ci demandaient à voir les dossiers de procédure, ils faisaient des photocopies et rendaient ensuite les dossiers . La collection est en train d'être digitalisée, car les photocopies perdent rapidement de leur netteté et sont en train de s'effacer. De nombreuses archives des etats permettent également de consulter des archives de l'époque de la dictature, liées à la police politique. Une partie du matériel disponible sont des reproductions de correspondances et des informations qui étaient transmis d'un État à l'autre, ou même à des agences comme le Service National D'Information (SNI). Les documents militaires sont cependant d'un accès très restreint, et dans beaucoup de cas, on se demande s'ils ont été détruits, comme dans le cas des documents qui concernent la guérilla de l'Araguaia, qui sont encore à ce jour inconnus du public et des chercheurs.

Dans d'autres pays du cône Sud, la question est encore plus compliquée. En Argentine par exemple, il y a la Comisión Provincial por la Memoria de La Plata, qui abrite I'archive de la DIPBA:

"I'archive de la Direction des Renseignements de la Police de la Province de Buenos Aires (DIPBA) est un registre d'espionnage politique- 


\begin{abstract}
idéologique sur des hommes et des femmes, étendu et détaillé , s'étendant sur un demi-siècle. La DIPBA fut créé en août 1956 et fonctionna jusqu'à ce qu'elle soit supprimée le 10 août 1998, dans le contexte de la réforme de la police de la province de Buenos Aires, et que son archive soit fermée."

(http://www.comisionporlamemoria.org/archivo/?page_id=3 consulta em 28/03/2012).
\end{abstract}

Néanmoins, l'accès aux informations est très compliqué, elle se fait à travers de photocopies qui doivent être demandées à partir d'une sélection de documents effectuée par le fonctionnaire de cette archive à travers des mots-clés, qui ne peuvent pas être des noms de personnes.

D'un autre côté, il existe des archives différentes, issues de l'initiative de la société civile comme le centre de documentation et de recherche sur la culture des gauches en Argentine (CeDInCl ) (http://www.cedinci.org/ ), fondée par un militant est soutenue par une association, actuellement liée à l'université nationale de San Martin, et la Memoria Abierta, action coordonnée d'organisations Argentines des droits humains (http://www.memoriaabierta.org.ar) qui a pour objectif de recueillir la mémoire de l'époque de la dictature et des actions de résistance. Le CeDInCl conserve tout particulièrement des journaux et d'autres documents des organisations de gauche. À l'inverse, la Memória Abierta a une politique (de conservation) centrée sur l'histoire orale.

$\mathrm{Au} \mathrm{Chili,} \mathrm{ce} \mathrm{sont} \mathrm{également} \mathrm{les} \mathrm{organisations} \mathrm{non-gouvernementales} \mathrm{qui} \mathrm{se}$ chargent de préserver la mémoire sur la répression et la résistance. Le lieu où nous avons rencontré le plus de matériel dans ce pays fut la FLACSO, qui possède une bibliothèque et un fonds documentaire extrêmement important accessible en ligne, par le biais d'une inscription. Et dans la Vicaria de la Solidaridad, une organisation liée à l'église catholique qui prêtait une assistance sociale et juridique aux victimes de la répression et à leurs familles.

L'archive la plus étonnante que nous avons rencontré dans notre recherche, fut le Centro de Documentación y Archivo para la Defensa de los Derechos Humanos, qui se trouve dans le Museo de la Justicia, également connu comme I'“Archivo del Terror” qui conserve les restes d'un immense fond d'archives composé de rapports de surveillance 

personnes se distinguent des étudiants, des syndicalistes, des prêtres ou des soeurs, tout étranger qui arrivait au pays, les épouses et la famille de "suspects ", et beaucoup d'autres, y compris des «féministes ${ }^{6}$. Cette documentation a été découverte en 1992 après la chute du gouvernement de Stroessner, et l'organisation de cette archive et sa mise à disposition pour la consultation ont pu être effectués grâce au soutien d'un réseau international, incluant l'Unesco.

Une autre source très importante de données pour cette période sont les archives et les collections de documents conservés dans d'autres pays. C'est le cas des collections, comme la de la Bibliothèque Internationale de Documentation Contemporaine à I'Université de Paris X, Nanterre, ou comme la Collection de Pamphlets sur l'Amérique Latine de l'université de Princeton, qui a été microfilmée, et qui est disponible dans de nombreuses bibliothèques et à l'achat. Autre possibilité sont toujours les archives diplomatiques. J'ai eu l'opportunité de voir les archives diplomatiques de l'ambassade française au Brésil, a Nantes, et plus récemment dans les Archives Nationales des ÉtatsUnis, a College Park, la correspondance du Department d'État avec plusieurs ambassades a L'Amerique du Sud.

Nous avons donc, pour travailler sur cette époque des dictatures du cône Sud, trois types de documents principaux : la documentation des archives policières et judiciaires, qui ne sont pas toujours accessibles facilement, la documentation des archives construites par des organisations qui veulent dénoncer les atrocités commises par les dictatures, engagées dans la défense des droits humains, et quelques archives qui tentent de documenter l'action des groupes de gauches pendant cette période. En plus de cela, il y a les archives diplomatiques. Les caractéristiques de la documentation, la manière d'organiser les données et l'accès aux documents sont différents pour chacune de ces

\footnotetext{
6 J'ai rendu visite à ces archives avec Joana M. Pedro, Mirian A. Nascimento et Larissa M. Freitas en juillet 2010. http://www.pj.gov.py/contenido/132-informacion-general-museo2/334; http://www.unesco.org/webworld/paraguay/historia.html
} 
archives. Mais il est possible d'utiliser ces données de manière comparative, dans la mesure où l'on effectue une critique, toujours aussi importante, de chaque document : d'où vient ce document, comment il fut produit, pourquoi et comment il fut conservé dans cette archive.

Dans le cas de notre recherche, utiliser ces archives relève d'un très grand défi, puisqu'elles n'ont jamais été pensées pour une recherche qui se centre sur l'étude du genre dans le contexte des dictatures. Toutefois, il a été très intéressant de rencontrer dans ces diverses archives, des traces qui renseignent sur la manière dont les discours de genre furent utilisés autant par la répression que par la résistance.

Le concept de Résistance est très large. Généralement pensé par opposition au « pouvoir ", la résistance peut-être tout type d'action individuelle ou collective réalisée contre un gouvernement, une institution, une loi, une action répressive. Cette notion de résistance comme un large éventail d'action et de mouvements contre dictature, tyrannie, ou régime d'exception est utilisé par les historiens du monde entier, par les spécialistes des sciences sociales et par d'autres dans des contextes très diversifiés ${ }^{7}$. Il est appliqué tant pour les mouvements armés comme les Partisans en France, les mouvements de libération d'Algérie et de toute l'Afrique, que pour les mouvements pacifistes comme la lutte de Nelson Mandela contre l'Apartheid, en Afrique du Sud, ou les mouvements des droits humains aux États-Unis.

C'est pour cela que je pense que je peux utiliser cette notion de résistance, dans le contexte des dictatures du cône Sud, en y incluant la guérilla, les mouvements des droits de I'homme, et même les organisations des familles des prisonniers et disparus, tout comme d'autres types de mouvements, comme les associations professionnelles et les partis d'opposition. D'un autre côté, l'inclusion de toute cette large gamme de mouvements et de groupes, sous un même " toit ", comporte ses propres dangers, puisqu'ils sont très différents, et même parfois opposés sur le plan de leurs objectifs et de leurs stratégies. Dans ma perspective de recherche, toutefois, il existe une différence

\footnotetext{
7 RIDENTI, Marcelo. Resistência e mistificação da resistência armada contra a ditadura: armadilhas para pesquisadores. In: REIS, Daniel Aarão, RIDENTI, Marcelo e MOTTA, Rodrigo Patto Sá. (orgs.). O golpe e a ditadura militar: quarenta anos depois (1964-2004). Baurú, SP: Edusc, 2004., pp. 53-65, p. 54.
} 
cruciale : la forme d'utilisation du genre dans le discours. Les organisations armées ont fréquemment utilisé la masculinité comme un argument pour lutter contre la dictature. L'orgueil, I'honneur, l'action (versus la passivité), la force et la capacité de supporter tous les types de défi physique et moraux au nom d'un idéal, sont utilisés pour décrire comment un guérillero devrait être, et expliquer son action ${ }^{8}$. A l'inverse, le discours de dénonciation de la violence de la répression utilisa le genre d'une autre manière, quasiment posé. Un grand nombre de dénonciations dans les médias concernait l'usage de la torture. Un autre type de dénonciation concernait la disparition de militants, et insistait souvent sur le désespoir des mères et d'autres membres de la famille du fait de cette disparition. Un autre type mettait l'accent sur l'injustice de nombreux emprisonnements, puisque beaucoup de personnes prisonnières n'étaient pas des militants politiques. Dans tous les discours, le genre est un élément important.

\section{Mães Dolorosas (Mères souffrantes).}

En analysant les documents de ces organisations, nous percevons comment les émotions et les sentiments liés au genre et surtout à la maternité furent mobilisés pour sensibiliser l'opinion publique. Il paraît différent de dire qu'un militant ou un guerrillero avait été torturé et était disparu, ou qu'une mère dise : « je cherche mon fils ».

\footnotetext{
8 Pour Che Guevara: "The guerrilla combatant is a night combatant; to say this is to say at the same time that he must have all the special qualities that such fighting requires. He must be cunning and able to march to the place of attack across plains or mountains without anybody noticing him, and then to fall upon the enemy, taking advantage of the factor of surprise which deserves to be emphasized again as important in this type of fight. After causing panic by this surprise, he should launch himself into the fight implacably without permitting a single weakness in his companions and taking advantage of every sign of weakness on the part of the enemy. Striking like a tornado, destroying all, giving no quarter unless the tactical circumstances call for it, judging those who must be judged, sowing panic among the enemy combatants, he nevertheless treats defenseless prisoners benevolently and shows respect for the dead." GUEVARA, Ernesto Che. Guerrilla Warfare. P. 24.
} 


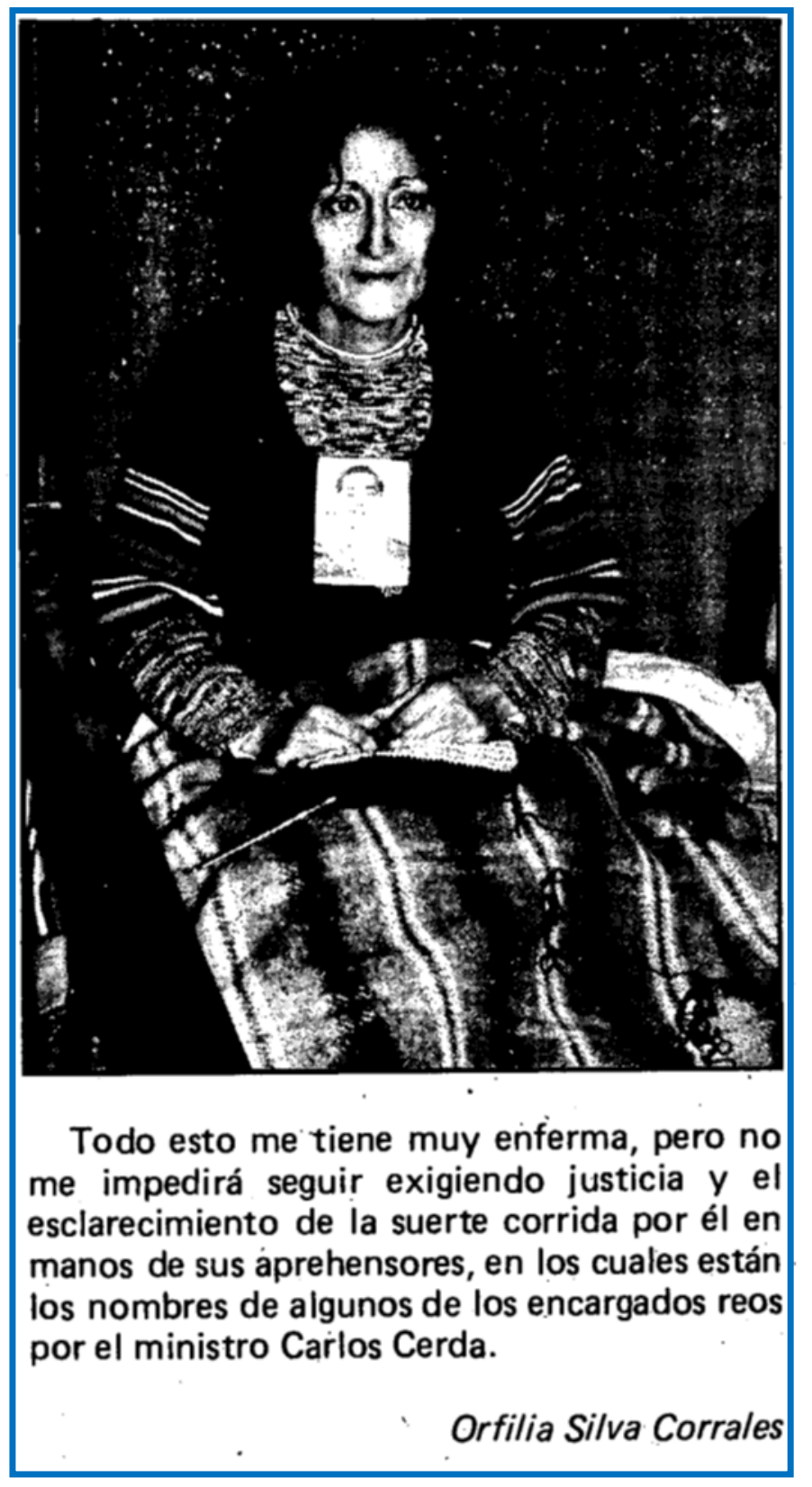

(Agrupación de Familiares de Detenidos Desaparecidos

Titulo: ¡Hasta encontrar la verdad! Detenidos - Desaparecidos, diciembre 1976, 1986 Colección: Fundación de Documentación y Archivo de la Vicaría de la Solidaridad, in: http://www.archivovicaria.cl/archivos/VS4b4f1f92850eb_14012010_1043am.pdf Consulté a 31/01/2011)

Cette image de la mère avec le portrait de son fils, est récurrente dans tous les documents. Elle a complètement symbolisé la dénonciation des atrocités commises dans plusieurs pays par les dictatures. 
Dans les archives qui conservent des documents liés aux droits de l'homme, les lettres des groupes de parents, de religieux et d'autres personnes sont des documents fréquents. Des pamphlets, des appels à la manifestation, des pancartes sont également présents massivement. D'un autre côté, cette même documentation se rencontre souvent dans les archives policières, ayant été confisquée lors de l'emprisonnement du militant ou fruit de la vigilance stricte exercée sur les personnes qui se risquaient à dénoncer les séquestrations, les tortures et les assassinats. L'association des mères de la Plaza de Mayo a été l'une des organisations qui a le plus mobilisé le discours de la maternité, et utilisé le genre comme une stratégie de protection, de dénonciation et de sensibilisation. Aujourd'hui, elle est divisée en deux organisations, les Madres de la Plaza de Mayo- Linea Fundadora (ligne fondatrice) et une autre du fait de divergences et de diverses questions. Le symbole maximum de cette association continue d'être le lange blanc (utilisé comme fichu) sur la tête de ces femmes qui demandent l'apparition de leurs enfants en vie ou la punition des responsables de leur assassinat.

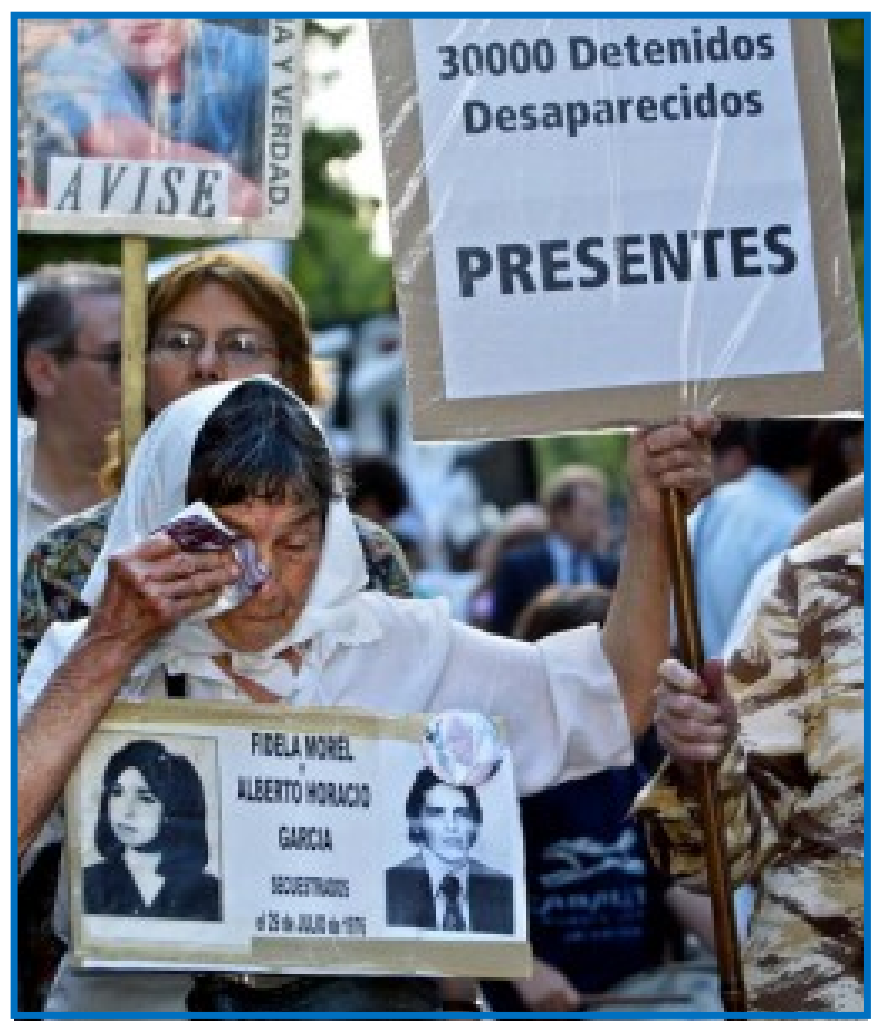

http://www.focoblanco.com.uy/2011/06/contra-las-madres-de-plaza-de-mayo/ 
Le lange fait référence aux soins que procurent les mères à leurs enfants. Elles utilisent stratégiquement leur rôle de mères, des mères comme celles qui soignent leurs enfants, liés à elles par un lien émotionnel très fort, afin d'humaniser les guérilleros et les militants de gauche. Plus que tout, avant d'être des guérilleros, ou des terroristes comme les gouvernements militaires les appelaient, ces jeunes étaient des fils et des filles, des bébés qui utilisaient des langes, qui ont reçu les soins de leur mère, des personnes, des humains. Leur discours est toujours exprimé sur ce ton émotionnel :

«Les Madres de Plaza de Mayo, nous continuerons à lutter, pas seulement avec la parole, mais aussi en exposant notre poitrine et notre tête chaque fois qu'il sera nécessaire, pour nos enfants, mais également pour tous les hommes et les femmes de cette terre qui souffrent l'injustice des oppresseurs qui se sont toujours cachés derrière leurs uniformes de garde. Et nous continuerons à lutter, parce que nous sommes toujours enceintes de nos enfants, c'est-à-dire de la libération et de l'espérance. 4 fevrier 1988 »9

Las Madres de Plaza de Mayo seguiremos luchando,
no solo con la palabra, sino poniendo el pecho y la ca-
beza cada vez que sea necesario, por nuestros hijos, pero
también por todos aquellos hombres y mujeres de esta
tierra qué sufren la injusticia de los opresores que sienl-
pre se esconden detrás de los uniformados de turno.
Y vamos a seguir luchando, porque nosotras esti-
mos embarazadas para siempre de nuestros hijos, que
es como decir de liberación y de esperanza.

4 de febrero de 1989

Carta Abierta de la Asociacion Madres de Plaza de Mayo al Dr. Raul Alfonsin. 4/02/1988. Buenos Aires, Argentina. Human Rights in Argentina II - Panphlets. Princeton University Libraries, Princeton, 1991. Microfilm. Consulted in Mckeldin Library, UMD College Park.

\footnotetext{
${ }^{9}$ Carta Abierta de la Asociacion Madres de Plaza de Mayo al Dr. Raul Alfonsin. 4/02/1988. Buenos Aires, Argentina. Human Rights in Argentina II - Panphlets. Princeton University Libraries, Princeton, 1991. Microfilm. Consulted in Mckeldin Library, UMD College Park.
} 
Elles disent qu'elles seront toujours enceintes de leurs enfants, qui continuent à faire parti de leur vie, même après être morts. Comme le dit Chico Buarque, dans une autre chanson consacrée au sujet de la mère qui a un fils disparu, "la nostalgie est le revers d'un accouchement, la nostalgie consiste à ranger la chambre d'un fils qui est déjà mort" ${ }^{10}$. Mais ici, nous sommes seulement en train de signaler comment l'émotion et le sentiment, exposés publiquement, ont joué un rôle important dans la sensibilisation de I'opinion publique, et spécialement dans l'« humanisation » des militants, à partir de l'engagement de leurs mères. Et comment est-ce que ça apparait dans les archives sombres de cette époque.

\section{Torture}

Lynn Hunt, dans son livre sur l'histoire de l'idée des Droits Humains, montre comment la dénonciation des supplices et les tortures pratiquées dans le système pénal en France et dans les autres endroits du monde, fut importante pour la construction d'une opinion publique opposée à l'usage de ses moyens et pour la construction des déclarations et des lois qui ont institué les droits humains dans leur qualité de droit. En Amérique du Sud, dans les pays dont nous parlons, la dénonciation de la torture pratiquée systématiquement contre les militants des organisations de gauche et même des personnes qui parfois ne faisaient même pas parti de ces organisations, fut également extrêmement importante pour la conquête de changements démocratiques dans ces pays.

Il est vrai qu'il est difficile de rencontrer des références à la pratique de la torture dans les archives officielles. Elles ne mentionnent pas ces pratiques totalement illégales. D'un autre côté, dans les archives des organisations des droits de l'homme, il y a un grand

\footnotetext{
10 Chico Buarque de Hollanda, Pedaço de Mim, 1979. Opera do Malandro. Ludmila Catela a étudié dans sa thèse la manière dont les familles de disparus se reconstruisent après la perte de leurs fils, de leur fille, de leurs époux, et de leur frère. La dénonciation, la lutte pour les informations, la nécessité de rechercher des appuis auprès d'autres familles, auprès de l'église ou des organisations de droits de l'homme, conduit ces familles à réorganiser toute leur vie autour de ces personnes qui sont mortes, qui ont disparues, mais qui n'ont certainement pas été oubliées ${ }^{10}$. Un processus similaire fut analysé par Deusa Maria de Sousa sur un groupe de familles de disparus de I'Araguaia ${ }^{10}$.
} 
effort pour documenter ces pratiques sous des formes variées : photo, témoignages recueillis des personnes qui ont été prisonnières. Et dans certaines archives étrangères, il est possible de retrouver des mentions de cette pratique. C'est le cas par exemple du militant Rene Louis de Carvalho, du parti communiste brésilien révolutionnaire (Partido Comunista Brasileiro Revolucionário (PCBR). Fait prisonnier en 1970, sa mère, une française, ex militante de la résistance pendant l'occupation allemande de la France, René de Carvalho , a fait appel à l'ambassade de France. Comme il était un citoyen français, à l'inverse de son père et de son frère qui étaient également emprisonnés, l'ambassadeur français a pu lui rendre visite en prison.

La torture, humanise également les militants. Et le genre fut aussi très utilisé dans les dénonciations. D'une certaine manière, il semble que lorsque l'on parle de torture sur des femmes, sur des enfants, et spécialement sur des femmes enceintes, la dénonciation gagne du poids, elle devient plus grave, elle provoque plus d'émotion.

La dénonciation suivante concerne une femme, Veronica Handl, torturée alors même qu'elle était enceinte, Cette dénonciation exige sa libération et celle de son fils , Pablo, qui serait né en prison, en 1977, en Argentine. Tout le discours renvoie à un ton ému, qui invoque l'injustice de la prison et les conditions inhumaines de son emprisonnement : « nous invitons l'opinion publique internationale à demander au gouvernement argentin la liberté de cette femme innocente et de son fils, (qui souffrent) des conditions inhumaines de leur injuste emprisonnement" (traduction libre). 


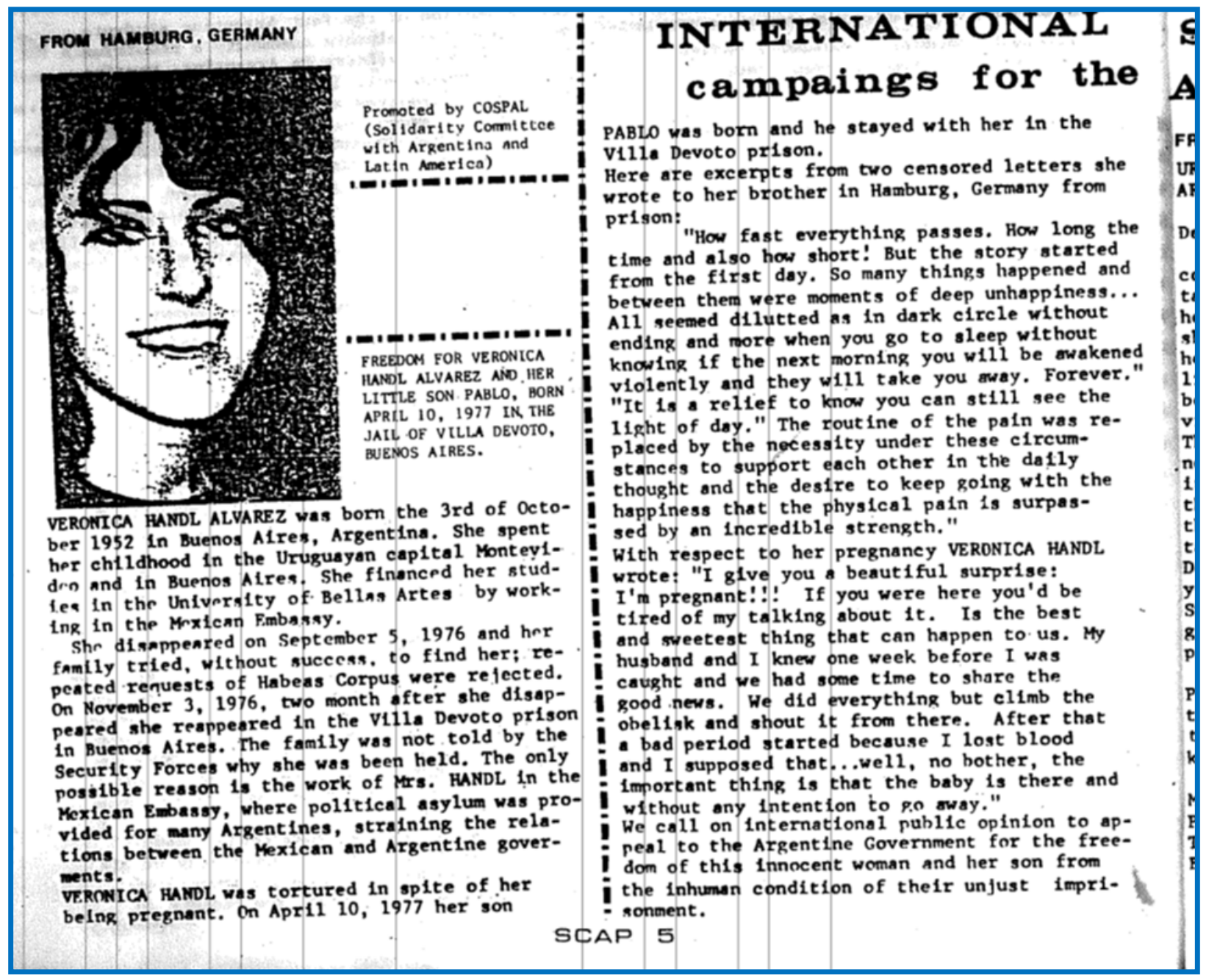

De la même manière, des cas comme ceux-ci furent dénoncés dans plusieurs lieux.

L'histoire de Maria Amelia Almeida Telles et de sa soeur Criméia, par exemple, sont des histoires qui ont été mentionnées à plusieurs reprises et qui ont toujours provoqué des sentiments d'injustice et d'indignation. Maria Amelia était une militante du Parti communiste du Brésil (PCdoB). Elle a travaillé notamment dans l'écriture, la composition et l'impression du journal du parti à Sao Paulo où elle a été arrêté en 1972. Criméia était une infirmière, elle a travaillé pendant un certain temps dans l'Araguaia, avec le groupe appelé plus tard la Guerrilla do Araguaia. Lorsqu'elle était enceinte, elle est venue à Sao Paulo, afin d' établir des contacts pour le groupe. L'arrestation de Maria Amélia et de toute sa famille, y compris de sa fille de six ans Janaina et de son fils Edson, 4 ans, de son mari, César, et de sa sœur, a eu 
pour objectif de chercher à identifier les leaders du PCdoB et en particulier les éléments de liaison entre le groupe Araguaia avec le parti.

Maria Amelia a été très torturée et elle a soutenu pendant un certain temps la version que sa sœur était une servante de la maison, en essayant de gagner du temps. Comme Criméia était enceinte de 8 mois et que César avait la tuberculose et le diabète, Amelia a souvent été torturée en face d'eux, afin qu'ils parlent. Les enfants sont restés pendant un certain temps, une ou deux semaines, dans la même prison, surveillés par la police. Ils ont parfois été amenés pour voir leur mère, ce qui générait du stress supplémentaire pour elle, surtout parce que la police lui proférait des menaces.

Elle a déclaré qu'une fois, lorsqu'elle était dans la chaise du dragon, ses enfants ont été amenés à la chambre de torture, et son fils lui a dit «maman, pourquoi papa est bleu et toi tu es verte? ». Elle s'est ensuite rendue compte qu'elle était toute couverte d'ecchymoses de couleur pourpre. Amelia et sa famille ont rapporté leur témoignage pour Brasil Nunca Mais, aux journaux, et les auditoriums etaient remplis (pour les écouter). Et dans un geste sans précédent, avec sa famille, elle a pu légalement dénoncer le colonel Ustra pour avoir ordonné la torture.

Si ces archives abritent ces mémoires dures, tristes, difficile, elles abritent également une histoire des personnes, des femmes et des hommes, qui n'ont jamais désisté, qui ont résisté, qui ont utilisé les armes de l'émotion, des sentiments, du genre, de la dénonciation pour construire un autre temps. Elles abritent une espérance. 
